\title{
Kick and Phase Errors in Spontaneous and Amplified
}

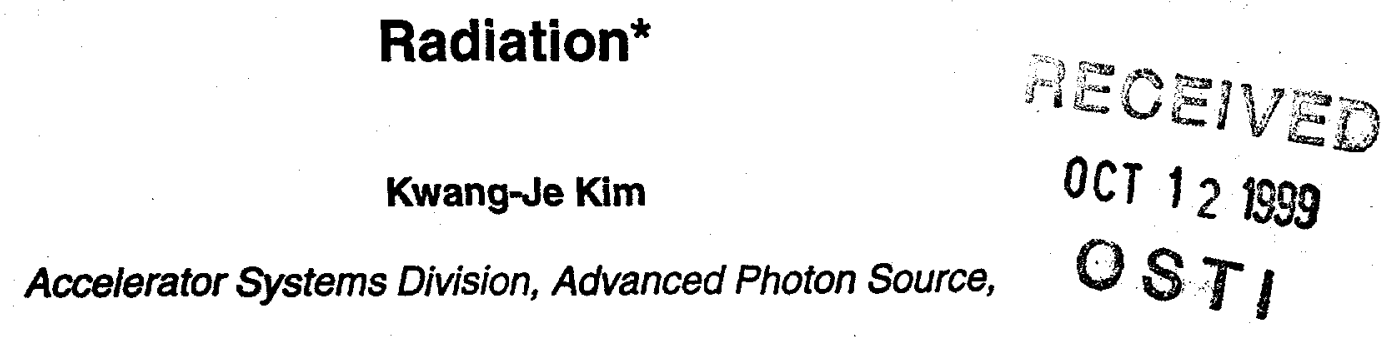

Argonne National Laboratory, 9700 S. Cass Avenue,

Argonne, IL 60439 U.S.A.

Phone: 630.252 .4647$, FAX: 630.252 .7369

E-mail: kwangje@aps.anl.gov

Two types of magnet errors are considered--the random phase error (RPE), in which the phase errors are evenly distributed along the magnet, and the random kick error (RKE), in which the errors in the derivative of the phase are evenly distributed. We compute the reduction in performance of both spontaneous radiation and high-gain free-electron lasers for both types of errors within the framework of 1-D free-electron laser theory.

Keywords:

Classification Codes:

The submitted manuscript has been created by the University of Chicago as Operator of Argonne National Laboratory ("Argonne") under Contract No. W-31-109-ENG-38 with the U.S. Department of Energy. The U.S. Government retains for itself, and others acting on its behalf, a paid-up, nonexclusive, irrevocable worldwide license in said article to reproduce, prepare derivative works, distribute copies to the public, and perform publicly and display publicly, by or on behalf of the Government.

*Work supported by the U.S. Department of Energy, Office of Basic Energy Sciences, under Contract No. W- 


\section{DISCLAIMER}

This report was prepared as an account of work sponsored by an agency of the United States Government. Neither the United States Government nor any agency thereof, nor any of their employees, make any warranty, express or implied, or assumes any legal liability or responsibility for the accuracy, completeness, or usefulness of any information, apparatus, product, or process disclosed, or represents that its use would not infringe privately owned rights. Reference herein to any specific commercial product, process, or service by trade name, trademark, manufacturer, or otherwise does not necessarily constitute or imply its endorsement, recommendation, or favoring by the United States Government or any agency thereof. The views and opinions of authors expressed herein do not necessarily state or reflect those of the United States Government or any agency thereof. 


\section{DISCLAIMER}

Portions of this document may be illegible in electronic image products. Images are produced from the best available original document. 


\section{Introduction}

Effects of undulator errors on radiation performance have been studied by several authors [1-10]. The errors are most conveniently characterized by the phase error $[6,8]$

$$
\delta \phi(\tau)=k \int \frac{d z}{v_{z 0}}\left[v_{z}-v_{z 0}\right]
$$

Here $\mathrm{k}=2 \pi \lambda, \lambda=$ radiation wavelength, $\mathrm{z}$ is the distance along the undulator axis, $v_{z 0}$ is the average $z$-velocity in the ideal undulator, $v_{z}$ is the average z-velocity in the presence of errors, $\tau$ is the scaled longitudinal coordinate,

$$
\tau=2 p k_{u} z=N / N_{G}
$$

where $\rho$ is the Pierce parameter [11], $k_{u}=2 \pi / \lambda_{u}, \lambda_{u}=$ undulator period length, $N=z / \lambda_{u}$ (the number of the undulator periods), and $N_{G}=1 / 4 \pi p$ is the number of undulator periods in one nominal gain length. For cases considered here, $\mathbf{N}_{\mathbf{G}} \gg 1$.

For well-optimized undulators corrected by suitably placed shims, the phase errors are distributed uniformly along the undulator with a constant rms value $\sigma_{\phi}$ [8-10]. This type of error will be referred to as the random phase error (RPE). In the presence of RPE, the intensity of the spontaneous radiation in the forward direction is reduced by the factor [8]

$$
\mathrm{R}=1-\sigma_{\phi}^{2}
$$

For long undulators, the errors could be controlled by steering corrections at regular intervals $[3,5,6,7]$. In these cases, the errors in the phase derivative, $d \delta \phi / d \tau$, rather than the phase errors themselves, are uniformly distributed along the undulator. For this type of error, which will be referred to as the random kick error (RKE), the reduction in the power growth of high-gain FELs was found to be [7]

$$
\mathrm{R}=1-\frac{2}{9} \mathrm{~W} \tau
$$

where

$$
W=\frac{1}{T}\left\langle\left|\int_{0}^{T} d \tau \frac{d \delta \phi}{d \tau}\right|^{2}\right\rangle
$$

Here the angular brackets denote ensemble average. 
In this paper we generalize the above results to others cases-the reduction in spontaneous radiation for RKE and the reduction in high-gain growth for RPE. The results are summarized in Table 1.

Table 1. Reduction due to magnet errors

\begin{tabular}{|l|c|c|}
\hline & Random Phase Error (RPE) & Random Kick Error (RKE) \\
\hline $\begin{array}{l}\text { Spontaneous radiation intensity in } \\
\text { the forward direction }\end{array}$ & $1-\sigma_{\phi}{ }^{2}$ & $1-\frac{1}{6} \mathrm{~W} \tau$ \\
\hline High-gain FEL growth rate & $1-\frac{\sigma_{\phi}{ }^{2}}{\sqrt{3}}$ & $1-\frac{2}{9} \mathrm{~W} \tau$ \\
\hline
\end{tabular}

\section{Error types}

In this section, we introduce mathematical models for the two types of errors. We suppose that the errors originate from discrete points (such as the magnet poles), $\tau_{j}=(\Delta \tau) j, j=0,1,2, \ldots$

\subsection{RPE}

The mathematical model for RPE is as follows:

$$
\phi(\tau)=\sum_{j} \varepsilon_{j} g\left(\tau-\tau_{j}\right)
$$

where $\left\{\varepsilon_{j}\right\}$ is a set of independent random numbers, and we have

$$
\left\langle\varepsilon_{\mathrm{i}} \varepsilon_{\mathrm{j}}\right\rangle=\varepsilon^{2} \delta_{\mathrm{ij}}
$$

The function $\mathrm{g}(\tau)$ is an even function of $\tau$ with a width $\sigma_{\tau}$, normalized as

$$
\int g^{2}(\tau) d \tau=1
$$

We will assume

$$
\sigma_{\tau} \leq \Delta \tau \leq 1 / \mathrm{N}_{\mathrm{G}} \ll 1
$$

The rms phase error $\sigma_{\phi}$ is given by

$$
\sigma_{\phi}^{2}=\frac{1}{\mathrm{~T}} \int_{0}^{\mathrm{T}}\left\langle\phi^{2}(\tau)\right\rangle \mathrm{d} \tau=\frac{\varepsilon^{2}}{\Delta \tau}
$$

For the calculation in section 3 , we will be dealing with the phase derivative

$$
\Delta(\tau) \equiv \frac{d \delta \phi}{d \tau}=\sum_{j} \varepsilon_{j} f\left(\tau-\tau_{j}\right), f(\tau)=d g / d \tau,
$$

and will need the following integrals: 


$$
\int_{-\infty}^{\infty} d \tau^{\tau} \int_{-\infty}^{\tau} d \tau^{\prime} f\left(\tau^{\prime}\right) f\left(\tau^{*}\right)\left[1, \tau^{*}, \tau^{\prime}+\tau^{\prime}\right]=\left[0, \frac{1}{2}, 0\right]
$$

\subsection{RKE}

With a set of random numbers $\left\{\varepsilon_{i}\right\}$ satisfying Eq. (7), the mathematical model for RKE can be defined as

$$
\frac{\mathrm{d} \delta \phi(\tau)}{\mathrm{d} \tau} \equiv \Delta(\tau)=\sum_{\mathbf{j}} \varepsilon_{\mathrm{j}} \mathrm{f}\left(\tau-\tau_{\mathrm{j}}\right) .
$$

However, the function $f(\tau)$ here is quite different from the case of RPE. It is an even function with normalization

$$
\int f(\tau) d \tau=1
$$

For $\tau_{\mathrm{n}} \leq \tau<\tau_{\mathrm{n}+1}$,

$$
\delta \phi(\tau)=\int_{0}^{\tau} f\left(\tau^{\prime}\right) d \tau^{\prime}=\sum_{j=1}^{n} \varepsilon_{j}
$$

In RKE, the error is conveniently characterized by the average value of the square of the phase error per gain length W given by Eq. (5) [7]:

$$
\mathrm{W}=\frac{1}{\mathrm{~T}}\left\langle(\delta \phi(\mathrm{T}))^{2}\right\rangle=\frac{\varepsilon^{2}}{\Delta \tau}
$$

\section{Calculation of Reduction Factors}

We follow closely the method in reference [7]. The 1-D free-electron laser (FEL) equation in the presence of the phase error can be written as

$$
\frac{d a}{d \tau}=-i \Theta+i \Delta(\tau) a, \frac{d \Theta}{d \tau}=P, \frac{d P}{d \tau}=-a
$$

Here $\mathrm{a}$ is the scaled field amplitude, and $\Theta$ and $\mathrm{P}$ are the collective variables representing the phase and momentum, respectively [11]. Note that the phase derivative $\Delta(\tau)=\mathrm{d} \delta \phi / \mathrm{d} \tau$ appears in the FEL equation as a $\tau$ dependent detuning.

In the absence of errors, $\Delta(\tau)=0$, the solution of Eq. (17) with initial conditions $a=0, \Theta_{0}=\Theta$, and $P=0$ at $\tau=0$, is given by

$$
a_{0}(\tau)=\frac{\Theta_{0}}{3} \sum_{j=1}^{3} \frac{e^{i \lambda_{j} \tau}}{\lambda_{j}}
$$


Here

$$
\left(\lambda_{1}, \lambda_{2}, \lambda_{3}\right)=\left(\frac{1-\sqrt{3} i}{2}, \frac{1+\sqrt{3} i}{2},-1\right)
$$

are the eigenvalues. This solution describes both the spontaneous emission for $\tau \ll 1$ and the high gain behavior for $\tau \geq 1$ with the leading growth rate $\lambda_{1}$.

Solving Eq. (17) in perturbation theory to second order in $\Delta$,

$$
a(\tau)=a_{o}(\tau)\left[1+i F_{1}(\tau)-F_{2}(\tau)\right]
$$

Here

$$
\begin{gathered}
F_{1}(\tau)=\frac{1}{a_{0}(\tau)} \int_{0}^{\tau} \Phi\left(\tau-\tau^{\prime}\right) \Delta\left(\tau^{\prime}\right) \mathrm{a}_{0}\left(\tau^{\prime}\right) \mathrm{d} \tau^{\prime}, \\
F_{2}(\tau)=\frac{1}{a_{0}(\tau)} \int_{0}^{\tau} \mathrm{d} \tau^{\prime} \int_{0}^{\tau^{\prime}} \mathrm{d} \tau^{\prime \prime} \Phi\left(\tau-\tau^{\prime}\right) \Phi\left(\tau^{\prime}-\tau^{\prime \prime}\right) \Delta\left(\tau^{\prime}\right) \Delta\left(\tau^{\prime \prime}\right) \mathrm{a}_{0}\left(\tau^{\prime \prime}\right) .
\end{gathered}
$$

The Green's function $\Phi$ is given by

$$
\Phi(\tau)=\frac{1}{3} \sum_{j=1}^{3} \mathrm{e}^{i \lambda_{j} \tau}
$$

Therefore

$$
\begin{gathered}
|\mathrm{a}(\tau)|^{2}=\mathrm{R}\left|\mathrm{a}_{\mathrm{o}}(\tau)\right|^{2}, \\
\mathrm{R}=1+\left\langle\left|\mathrm{F}_{1}(\tau)\right|^{2}\right\rangle-\left\langle\left(\mathrm{F}_{2}+\mathrm{F}_{2} *\right)\right\rangle .
\end{gathered}
$$

This is the general expression for the reduction factor.

For the error models discussed in the previous section, we insert Eq. (11) (or Eq. (13)) to obtain

$$
\begin{gathered}
\left\langle\left|\mathrm{F}_{1}(\tau)\right|^{2}=\varepsilon^{2} \sum_{\mathrm{j}}\left|\int_{-\infty}^{\infty} \Phi\left(\tau-\tau^{\prime}-\tau_{\mathrm{j}}\right) \mathrm{f}\left(\tau^{\prime}\right) \frac{\mathrm{a}_{\mathrm{o}}\left(\tau^{\prime}+\tau_{\mathrm{j}}\right)}{\mathrm{a}_{\mathrm{o}}(\tau)} \mathrm{d} \tau^{\prime}\right|^{2},\right. \\
\left\langle\mathrm{F}_{2}(\tau)\right\rangle=\varepsilon^{2} \sum_{\mathrm{j}} \int_{-\infty}^{\infty} \mathrm{d} \tau^{\prime} \int_{-\infty}^{\tau^{\prime}} \mathrm{d} \tau^{\prime \prime} \Phi\left(\tau-\tau^{\prime}-\tau_{\mathrm{j}}\right) \Phi\left(\tau^{\prime}-\tau^{\prime}\right) \mathrm{f}\left(\tau^{\prime}\right) \mathrm{f}\left(\tau^{\prime \prime}\right) \mathrm{a}_{\mathrm{o}}\left(\tau_{\mathrm{j}}+\tau^{\prime \prime}\right) \mathrm{a}_{\mathrm{o}}{ }^{-1}(\tau) .
\end{gathered}
$$

Since the function $f(\tau)$ vanishes outside a small region $|\tau| \leq \sigma_{\tau}<<1$, the integrands in the above can be expanded in $\tau^{\prime}$ and $\tau^{\prime \prime}$. Let us consider various cases.

For spontaneous emission $\tau, \tau_{\mathrm{j}}, \tau^{\prime}$, and $\tau^{\prime \prime}$ are all much smaller than unity. Since $\mathrm{a}_{0}(\tau)=i \Theta \tau+0\left(\tau^{4}\right)$ and $\Phi(\tau)$ $=1+0\left(\tau^{3}\right)$ for $\tau \ll 1$, we obtain 


$$
R=1+\varepsilon^{2} \sum_{j}\left\{\left.\iint \tau^{\prime} f\left(\tau^{\prime}\right) \frac{\left(\tau^{\prime}+\tau_{j}\right)}{\tau}\right|^{2}-2 \int_{-\infty}^{\infty} d \tau^{\prime} \int_{-\infty}^{\tau^{\prime}} d \tau^{\prime} f\left(\tau^{\prime}\right) f\left(\tau^{\prime}\right) \frac{\left(\tau_{j}+\tau^{\prime}\right)}{\tau}\right\}
$$

For RPE, the first term in the curly bracket of Eq. (28) gives a negligible contribution, and the second term can be evaluated making use of Eq. (12). The result is

$$
R=1-\varepsilon^{2} \sum_{j=1}^{N} \frac{1}{\tau}=1-\frac{\varepsilon^{2} N}{\tau}=1-\sigma_{\phi}^{2}
$$

For $R K E, f(\tau)$ is an even function normalized by Eq. (14). Therefore

$$
R=1+\varepsilon^{2} \sum_{j}\left\{\left(\frac{\tau_{j}}{\tau}\right)^{2}-\left(\frac{\tau_{j}}{\tau}\right)\right\}=1-\frac{\varepsilon^{2} N}{6}=1-\frac{W \tau}{6}
$$

Equations (29) and (30) give the first row of Table 1.

In the exponential gain regime, the leading behavior is $a_{0}(\tau) \rightarrow\left(\Theta_{0} / 3 \lambda_{1}\right) e^{i \lambda_{1} \tau}, \Phi \rightarrow(1 / 3) e^{i \lambda_{1} \tau}$. Equation (25) is then easily evaluated:

$$
\begin{aligned}
\left|F_{1}(\tau)\right|^{2} & =\frac{N \varepsilon^{2}}{9} \int f\left(\tau^{\prime}\right) d \tau^{\prime} \\
& =0 \text { for RPE, } \\
& =\frac{N \varepsilon^{2}}{9} \text { for RKE. }
\end{aligned}
$$

In calculating $F_{2}$ from Eq. (27), we can replace the functions $\Phi\left(\tau-\tau^{\prime}-\tau_{j}\right), a_{0}\left(\tau_{j}+\tau^{\prime \prime}\right)$, and $a_{0}(\tau)$ in the integrand by the leading exponential behavior. However, the function $\Phi\left(\tau^{\prime}-\tau^{\prime}\right)$ must be evaluated exactly since $\mid \tau^{\prime}-$ $\tau^{\prime \prime} k<1$. We obtain

$$
\left\langle F_{2}(\tau)\right\rangle=\frac{N \varepsilon^{2}}{9} \sum_{n=1}^{3} \int_{-\infty}^{\infty} d \tau^{\prime} \int_{-\infty}^{\tau^{\prime}} d \tau^{\prime} \mathrm{e}^{\mathrm{i}\left(\lambda_{1}-\lambda_{\mathrm{n}}\right)\left(\tau^{\prime}-\tau^{\prime}\right)} \quad \mathrm{f}\left(\tau^{\prime}\right) \mathrm{f}\left(\tau^{\prime}\right)
$$

The function exp $i\left(\lambda_{1}-\lambda_{n}\right)\left(\tau^{\prime}-\tau^{\prime \prime}\right)$ can be expanded to $1+i\left(\lambda_{1}-\lambda_{n}\right)\left(\tau^{\prime}-\tau^{\prime \prime}\right)$, and the integral can then be evaluated using the properties of $f(\tau)$ discussed in Section 2 . In this way one obtains

$$
\begin{aligned}
\left\langle F_{2}(\tau)\right\rangle & =\frac{N \varepsilon^{2}}{2 \sqrt{3}} \quad \text { for RPE } \\
& =\frac{N \varepsilon^{2}}{6} \quad \text { for RKE. }
\end{aligned}
$$


Collecting these results, we obtain

$$
\begin{aligned}
\mathbf{R} & =1-\frac{\sigma_{\phi}{ }^{2}}{\sqrt{3}} \tau \quad \text { for RPE} \\
& =1-\frac{2}{9} N \varepsilon^{2}=1-\frac{2}{9} W \tau \text { for RKE. }
\end{aligned}
$$

Equation (33) reproduces the second row of Table 1.

\section{Discussion}

We have computed the reduction in the spontaneous intensity and in the high-gain growth for two types of errors, RPE and RKE. In the case of RKE, the average value of the square of the phase error per gain length W was used as the measure of the error magnitude rather than the rms phase error $\sigma_{\phi}$ that was used in the case of RPE. It is possible to introduce $\sigma_{q}$ for RKE by, for example,

$$
\sigma_{\phi}^{2}=\left\langle\frac{1}{N} \sum_{n}\left(\delta \phi_{n}-\xi_{n}\right)^{2}\right\rangle .
$$

Here, $\delta \phi_{n}$ is the phase error for $\tau_{n} \leq \tau \leq \tau_{n+1}$ given by Eq. (15). The parameter $\xi$ is determined by minimizing Eq. (34). One then obtains $\sigma_{\phi}^{2}=W \tau / 10$.

\section{References}

[1] B.M. Kincaid, J. Opt. Soc. Am. B2 (1985) 1294.

[2] C.J. Elliott and B. McVey, Proc. Adriatico Research Conference, Undulator Magnets for Synchrotron Radiation and FELs, Trieste, June 1987, World Scientific (1988) 143.

[3] H.D. Shay and E.T. Scharlemann, Nucl. Instr. Meth. Phys. Res. A272 (1988) 601.

[4] B. Diviacco and R.P. Walker, Proc. 1989 U.S. Particle Accelerator Conference, IEEE Conference Record 89CH2669-0 (1989) 1259.

[5] E. Esarey et al., Nucl. Instr. Meth. Phys. Res. A296 (1990) 423.

[6] B.L. Bobbs et al, ibid. 574.

[7] L.H. Yu et al., Phys. Rev. A45 (1992) 1163.

[8] R.P. Walker, Nucl. Instr. Meth. A335 (1993) 328.

[9] B. Diviacco and R.P. Walker, Nucl. Instr. Meth. A386 (1996) 522.

[10] R. Dejus et al., Rev. Sci. Instr. 66 (1995) 1875. 
R. Bonifacio, C. Pellegrini, and L.N. Narducci, Opt. Commun. 50, (1984) 373. 